\title{
Supporting Information \\ Large effective slip on lubricated surfaces measured with colloidal probe AFM
}

\author{
Liam R. J. Scarratt ${ }^{1, a}$, Liwen Zhu ${ }^{1}$, Chiara Neto ${ }^{1 *}$
}

1. School of Chemistry and the University of Sydney Nano Institute, The University of Sydney, New South Wales 2006, Australia.

a. Department of Inorganic and Analytical Chemistry, University of Geneva, Sciences II, Quai-Ernest-Ansermet 30, 1205 Geneva, Switzerland.

E-mail: chiara.neto@sydney.edu.au

Keywords: Slip length, Hydrodynamic Drainage Force, SLIPS, Colloidal Probe AFM, Lubricant Infused 
(a)

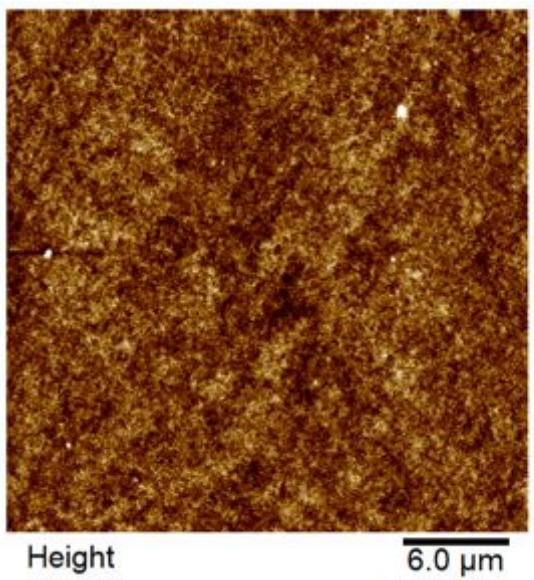

(b)

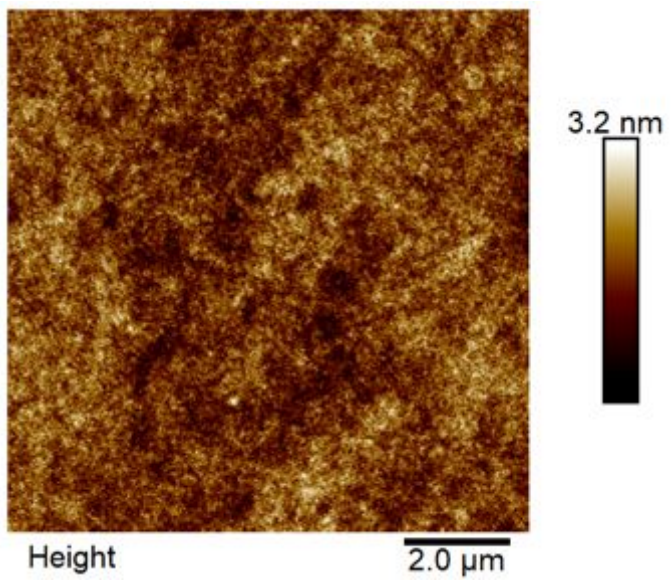

Figure S1. Tapping mode AFM micrographs of Teflon coated OTS-Si. RMS $0.46 \pm 0.04 \mathrm{~nm}$ from multiple measurements. 

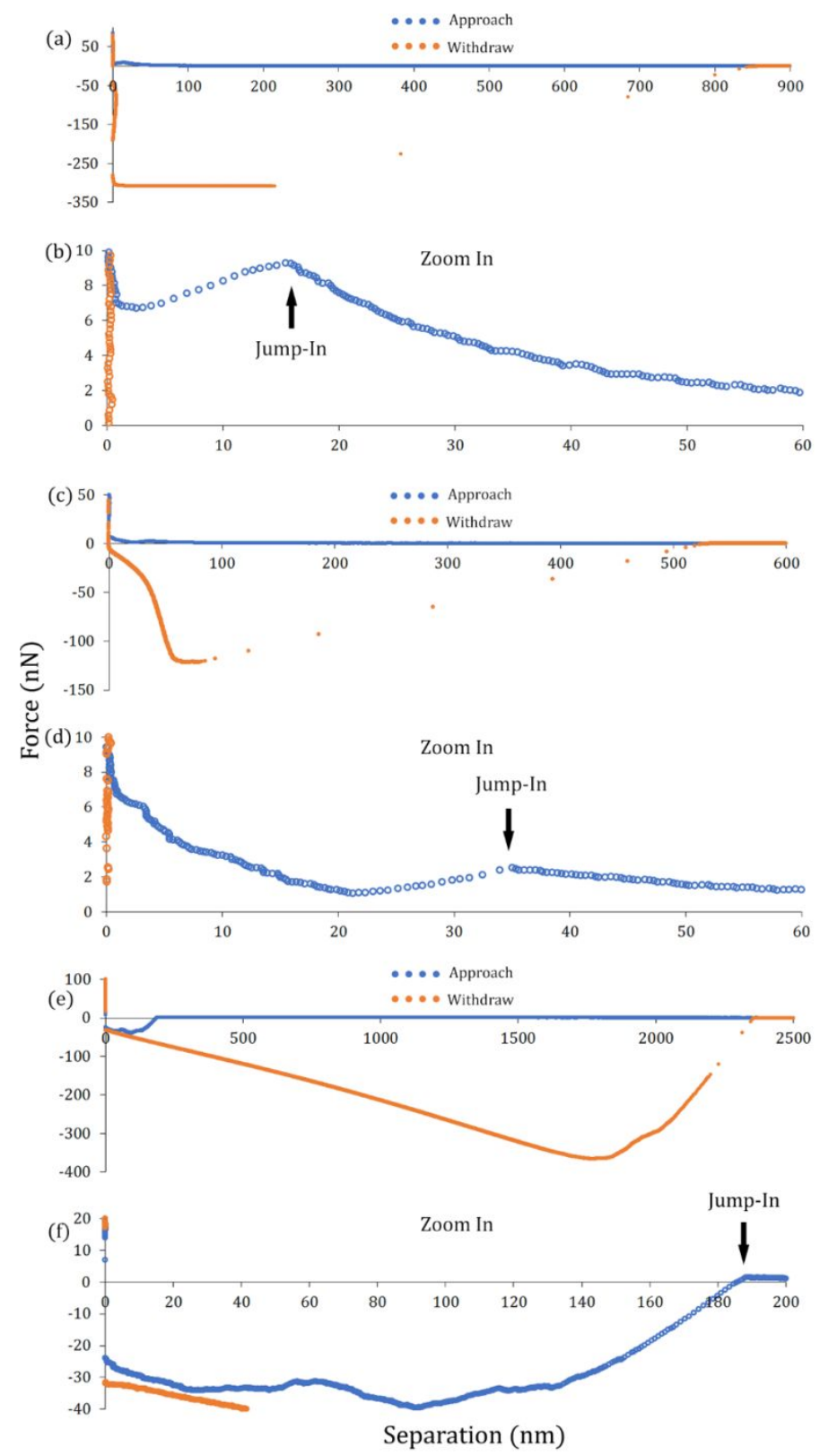

Figure S2. Determining silicone oil film thickness on Teflon in sucrose solution via meniscus force measurements, using colloid probe AFM. Distance between jump-in and hard contact during approach is used as film thickness (black arrows). Force curves over three different experiments are shown ((a), (c) and (e)) with a zoom in ((b), (d) and (f)) to highlight the jump-in at close separation. Experimental parameters are as follows: (a) and (b) $\mathrm{k}=0.53 \mathrm{~N} / \mathrm{m}$, radius $9.58 \mu \mathrm{m}$, approach rate $1 \mu \mathrm{m} / \mathrm{s}$. (c) and (d) $\mathrm{k}=0.3 \mathrm{~N} / \mathrm{m}$, radius $8.48 \mu \mathrm{m}$, approach rate $1 \mu \mathrm{m} / \mathrm{s}$. (e) and (f) $\mathrm{k}=0.94 \mathrm{~N} / \mathrm{m}$, radius $9.3 \mu \mathrm{m}$, approach rate $500 \mathrm{~nm} / \mathrm{s}$. 
(a)

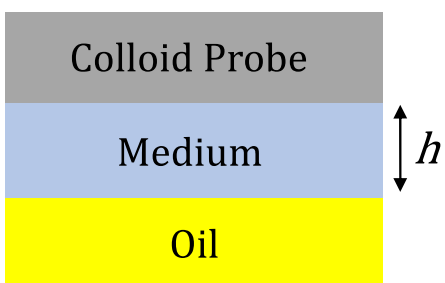

(b)

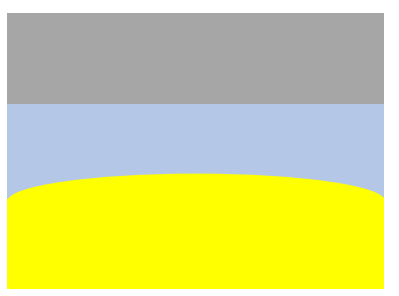

Figure S3.(a) Visualization of the three layer system used for Hamaker constant, $A$, calculations with lubricant (silicone oil) and colloid probe separated by medium (sucrose solution or water) with thickness $h$. (b) Upward deformation of the oil layer due to attractive van der Waals forces from the approaching colloid probe.

The non-retarded Hamaker constant $A$ for the three layer system of silicone oil/sucrose solution/silica colloid probe of $2.37 \times 10^{-21} \mathrm{~J}$, was calculated via Lifshitz theory: ${ }^{1}$

$$
A=\frac{3}{4} k_{B} T\left(\frac{\varepsilon_{1}-\varepsilon_{3}}{\varepsilon_{1}+\varepsilon_{3}}\right)\left(\frac{\varepsilon_{2}-\varepsilon_{3}}{\varepsilon_{2}+\varepsilon_{3}}\right)+\frac{3 \pi \hbar v_{e}}{4 \sqrt{2}} \times \frac{\left(n_{1}^{2}-n_{3}^{2}\right)\left(n_{2}^{2}-n_{3}^{2}\right)}{\sqrt{\left(n_{1}^{2}+n_{3}^{2}\right)\left(n_{2}^{2}+n_{3}^{2}\right)}\left[\sqrt{\left(n_{1}^{2}+n_{3}^{2}\right)}+\sqrt{\left(n_{2}^{2}+n_{3}^{2}\right)}\right]}
$$

Where $v_{e} \approx 4 \times 10^{15} \mathrm{~s}^{-1}$ is the plasma frequency of free electron gas, $k_{B}$ is the Boltzmann constant, $T$ is the absolute temperature, $\hbar$ is the reduced Plank constant, and $\varepsilon_{123}$ and $n_{123}$ are the dielectric constants and refractive indices of the tip, silicone oil lubricant, and liquid medium respectively.

\section{Estimate of errors in film thickness by meniscus force measurements}

To estimate the effects of van der Waals forces on the overestimation of the film thickness the following equations can be used. The separation distance, $H$, at which the cantilever experiences an attractive jump-in is given by Equation S1.

$$
H=(R A / 3 k)^{1 / 3}
$$


Where $A$ is the non retarded Hamaker constant $(\mathrm{J})$ for a three-layer system using the Liftshitz approximation, ${ }^{2}$ including the liquid film and cantilever tip separated by chosen medium, $R$ is the radius of the cantilever tip $(\mu \mathrm{m})$, and $k$ is the spring constant of the cantilever $(\mathrm{N} / \mathrm{m})$. To estimate the thickness of deformation of the liquid film due to attractive van der Waals forces towards the cantilever tip, $H_{f}$, Equation $\mathrm{S} 2$ can be used.

$$
H_{f}=(R A / 3 \pi \gamma)^{1 / 3}
$$

Where $\gamma$ is the surface tension of the liquid film in the chosen medium. In calculating the Hamaker constant, $A$, for a three layer system (silicone oil/sucrose solution/silica) as described above, the strength of the van der Waals forces between the substrate (Teflon/OTS-Si) and silicon oil film was not considered. The substrate effect would be more important in thinner films as the van der Waals forces between liquid film and substrate decay over distance. ${ }^{1}$

The maximum overestimation from cantilever jump-in due to attraction to the film (Equation $\mathrm{S} 1, A=2.4 \times 10^{-21} \mathrm{~J}, R=8.48-9.3 \mu \mathrm{m}$ and $\left.k=0.3-0.94 \mathrm{~N} / \mathrm{m}\right)$ is $\approx 2.8 \mathrm{~nm}$. The maximum overestimation due to deformation of the film due to attraction towards the tip (Equation S2) is $\approx 4 \mathrm{~nm}$. Therefore, there is a potential to overestimate the film thicknesses obtained with meniscus force measurements by up to $4 \mathrm{~nm}$. 

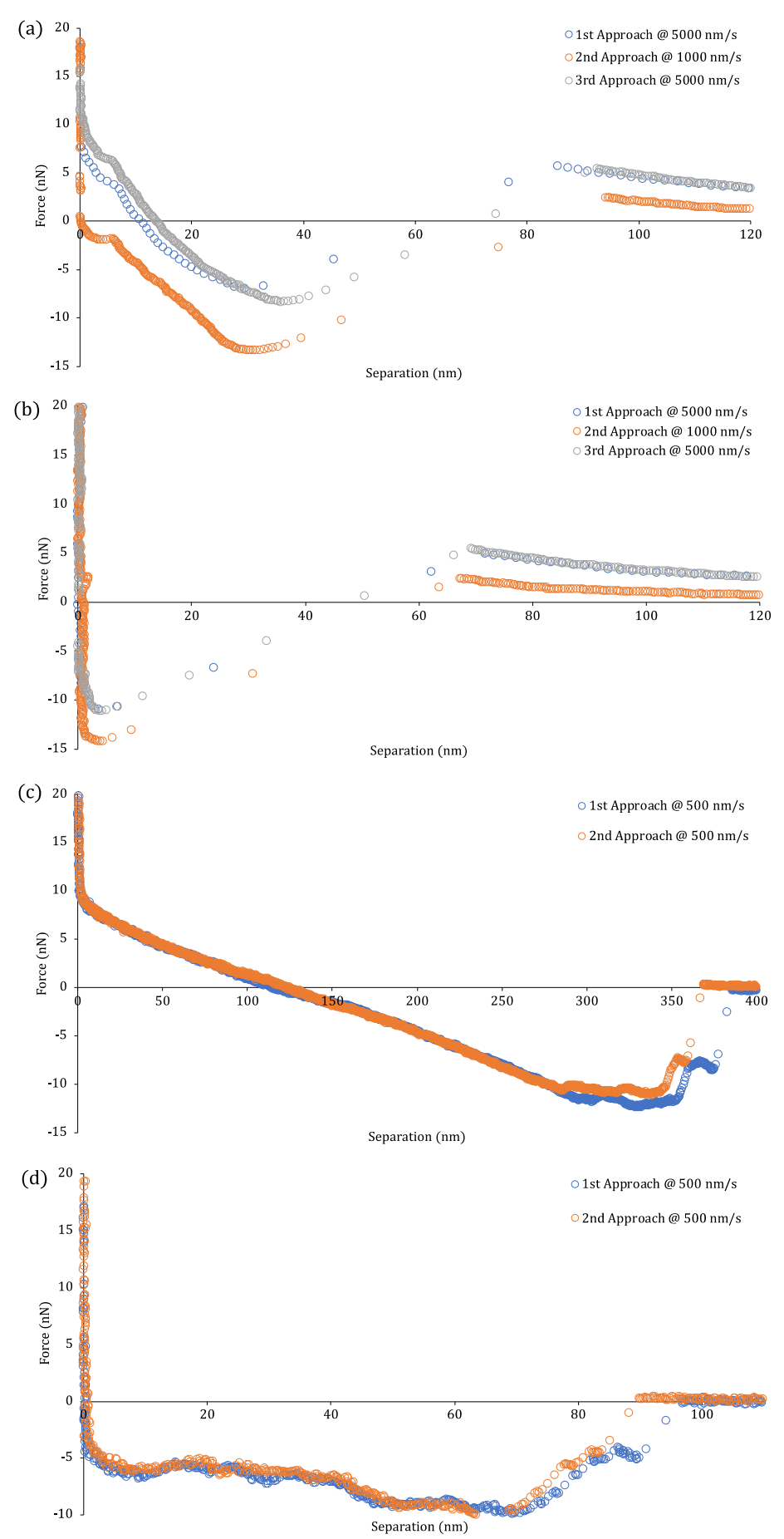

Figure S4. Determining silicone oil film thickness on Teflon in sucrose solution via colloid probe meniscus force measurements. Force curves over four different experiments are shown to highlight the variation of jump-in at close separation that occurs with repeated measurements with and without varying the approach rate. The order of the approach rate used is indicated in the legend of each panel. Experimental parameters are as follows: (a) and (b) $\mathrm{k}=0.3 \mathrm{~N} / \mathrm{m}$, radius $8.48 \mu \mathrm{m}$. (c) and (d) $\mathrm{k}=1.2 \mathrm{~N} / \mathrm{m}$, radius $9.3 \mu \mathrm{m}$. (a) Repeatability in film thickness 
obtained is generally good when changing approach rates. The point of jump-in at approach rate of $5 \mu \mathrm{m} / \mathrm{s}$ is within $5-10 \mathrm{~nm}$ of the first measurement at the same rate, after using an intermediate approach of $1 \mu \mathrm{m} / \mathrm{s}$. (b) Another example of good repeatability of film thickness, where the position of the jump-in in the $1^{\text {st }}$ and $3^{\text {rd }}$ approach at $5 \mu \mathrm{m} / \mathrm{s}$ are similar. The jump-in at the lower velocity occurs at a slightly lower silicone oil thickness. In (c) and (d) repeat measurements were performed at $500 \mathrm{~nm} / \mathrm{s}$ approach rates, with the $2^{\text {nd }}$ approach presenting a lower silicone oil thickness via the position of the jump-in. Here we see an error in jump-in position in of $17 \mathrm{~nm}$ in (c) and $6 \mathrm{~nm}$ in (d). Based on the data presented in (a) and (b), we suspect that further measurements over the same position at the same rate will not consistently produce lower values in cantilever jump in. 


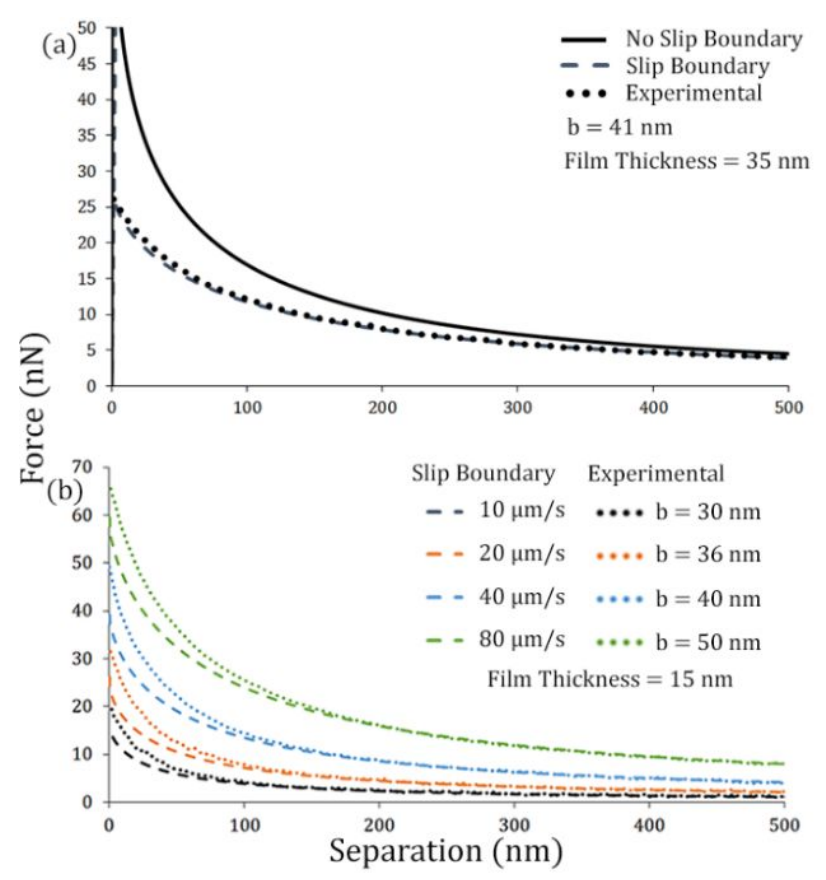

Figure S5. Hydrodynamic force measurements over thin silicone oil film on Teflon using colloidal probe AFM. (a) Single force curve compared to slip and no-slip boundary conditions with effective slip length of $41 \mathrm{~nm}$ fitted over a film thickness of $35 \mathrm{~nm}(\mathrm{k}=0.3 \mathrm{~N} / \mathrm{m}$, radius $8.48 \mu \mathrm{m}$, approach rate $40 \mu \mathrm{m} / \mathrm{s}$ ). (b) Multiple force curves over the same position at different approach velocities with corresponding slip boundary and effective slip lengths fitted over a film thickness of $15 \mathrm{~nm}(\mathrm{k}=0.94 \mathrm{~N} / \mathrm{m}$, radius $9.3 \mu \mathrm{m})$. 


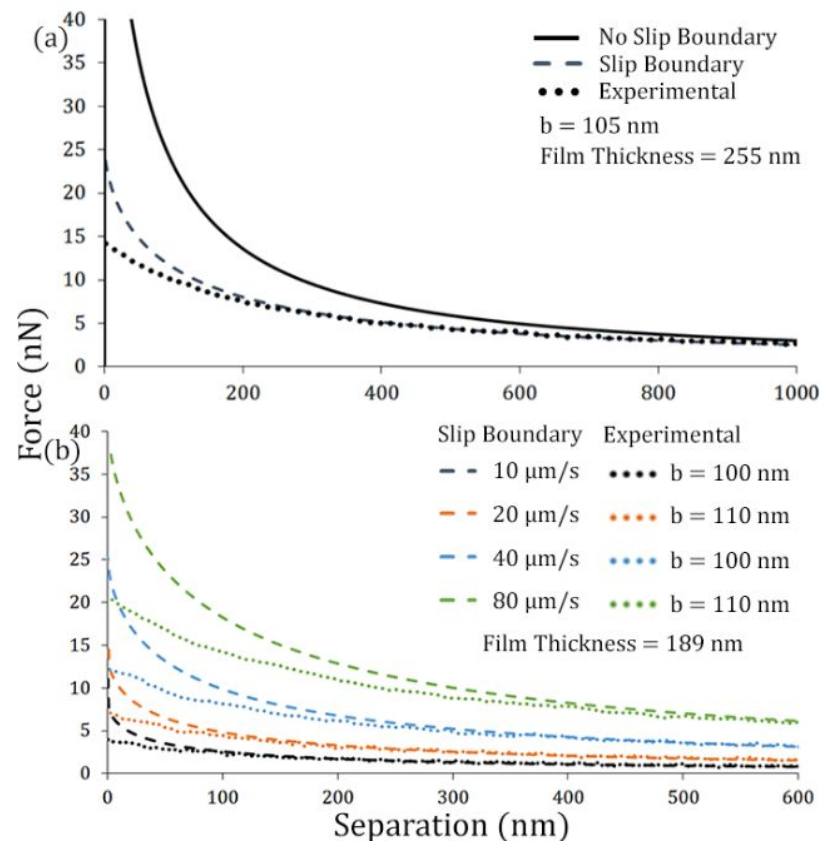

Figure S6. Hydrodynamic force measurements over thick silicone oil film on Teflon using colloidal probe AFM. (a) Single force curve compared to slip and no-slip boundary conditions with effective slip length of $105 \mathrm{~nm}$ fitted over a film thickness of $255 \mathrm{~nm}(\mathrm{k}=0.53 \mathrm{~N} / \mathrm{m}$, radius $9.58 \mu \mathrm{m}$, approach rate $40 \mu \mathrm{m} / \mathrm{s}$ ). (b) Multiple force curves over the same position at different approach velocities with corresponding slip boundary and effective slip lengths fitted over a film thickness of $189 \mathrm{~nm}(\mathrm{k}=0.94 \mathrm{~N} / \mathrm{m}$, radius $9.3 \mu \mathrm{m})$.

The increase in slip length with increasing approach velocity is not a reliable effect, as appears from comparing Figures S5 and S6. As long as the lubricant is not sheared away from the surface, ${ }^{3,4}$ it is possible for drag reduction to increase with increasing shear rate. ${ }^{5}$ However, fitting an accurate effective slip length over a deformable interface while increasing approach rate is challenging particularly with thicker films which deform more than thin films. 


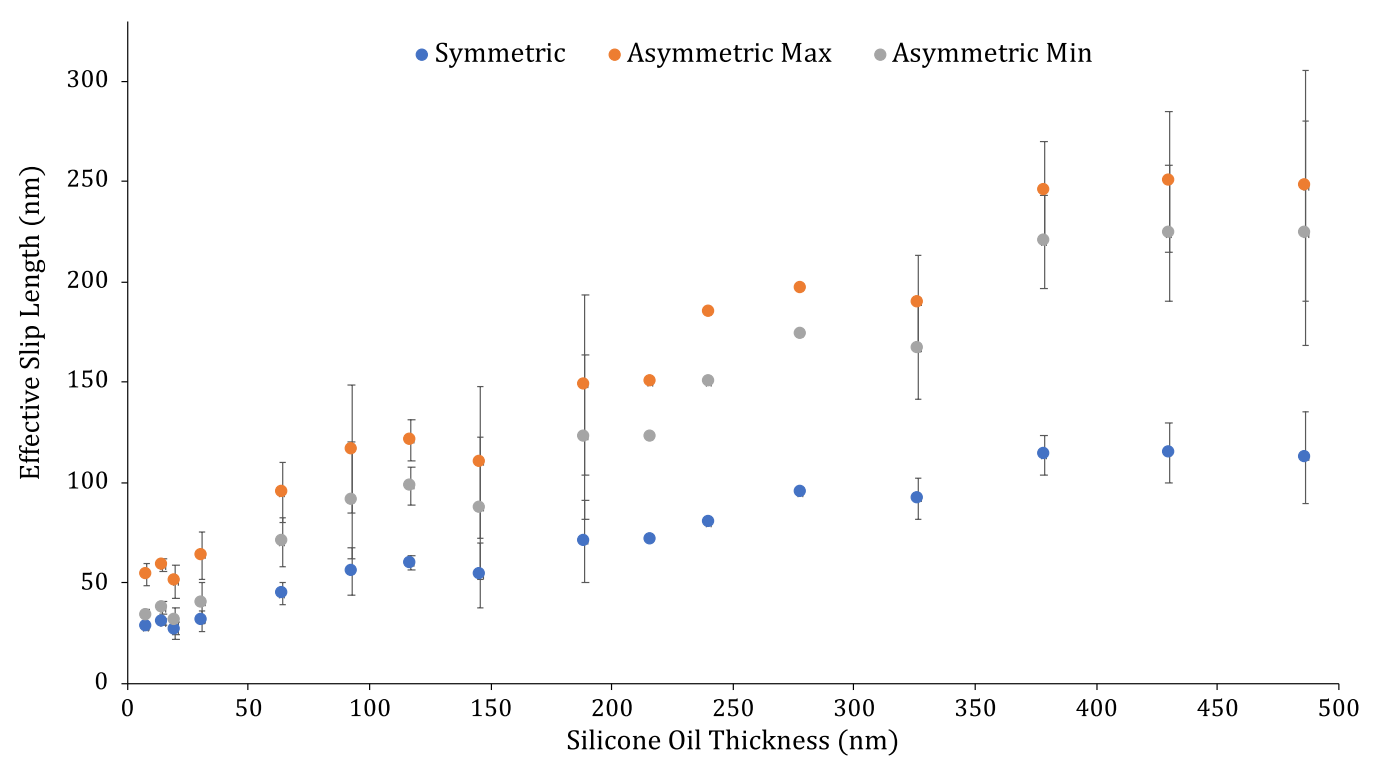

Figure S7. Effective slip length values obtained by fitting experimental data with the symmetric (blue dots) and asymmetric model (orange and grey dots) over different silicone oil film thickness at an approach rate of $10 \mu \mathrm{m} / \mathrm{s}$, plotted against corresponding film thickness $(\mathrm{k}=0.25$ $-1.2 \mathrm{~N} / \mathrm{m}$, radius $8.48-9.58 \mu \mathrm{m})$. Asymmetric max and min values were obtained by treating the effective slip length of the colloidal probe surface as $7 \mathrm{~nm}$ (for a clean silica sphere) and 24 $\mathrm{nm}$ (for a silicone-oil coated sphere), respectively.

Vinogradova's correction factor $f^{*}$ for the symmetric model of slip length is defined as: ${ }^{6}$

$$
f^{*}=\frac{h}{3 b}\left[\left(1+\frac{h}{6 b}\right) \ln \left(1+\frac{6 b}{h}\right)-1\right]
$$

When fitting an effective slip length, the symmetric slip model can be used for simplicity, as it assumes that both the surface of the colloid probe and the surface being studied have the same slip length. ${ }^{7}$ Using the asymmetric model takes into account the slip lengths of both surfaces, and results in larger effective slip length values over the surface of interest. For a smooth hydrophobic surface, this can increase the effective slip length from $25 \mathrm{~nm}$ to $44 \mathrm{~nm} .{ }^{8}$ The quality of the fit and shape of the hydrodynamic force vs separation does not vary significantly between symmetric and asymmetric models. 


\section{Effect of approach rates on measuring silicone oil thickness in meniscus force measurements}

When measuring silicone oil film thickness by AFM meniscus force measurements with a pointed tip in air, we previously found that the quantification of silicone oil film thickness was weakly dependent on approach rate. ${ }^{9}$ An approach rate lower than $100 \mathrm{~nm} / \mathrm{s}$ resulted in an overestimation of the silicone oil thickness, as the tip is attracted to the meniscus before it has reached the liquid film interface. However, at approach rates between $1000 \mathrm{~nm} / \mathrm{s}$ and $100 \mathrm{~nm} / \mathrm{s}$ the measured values of jump-in thickness were within error, so approach rates above $500 \mathrm{~nm} / \mathrm{s}$ were used for reliable silicone oil thickness measurements.

In this work, we also identified the ideal approach rate to measure the silicone oil film thickness. In this case we had to account for two competing effects: on the one hand, the previously observed effect of overestimation of silicone oil thickness at lower approach rates; on the other hand, given that our force curves are measured in a viscous liquid with a large colloid probe, we cannot ignore hydrodynamic forces that act to thin the silicone film thickness at higher approach rates. We have measured values of jump-in thickness at different approach rates to identify the rate at which the two effects balance out. Figure S8 shows two sets of force curves, with the sets in (a) and (b) taken over two different sample positions; here it can be seen that the value of jump-in separation increases gradually and slightly as the approach rate decreases from $1000 \mathrm{~nm} / \mathrm{s}$ to $100 \mathrm{~nm} / \mathrm{s}$. There are a number of values of jump-in separation that are closer to each other (are clustered within error, as observed in reference 9) and these are highlighted with a box. 

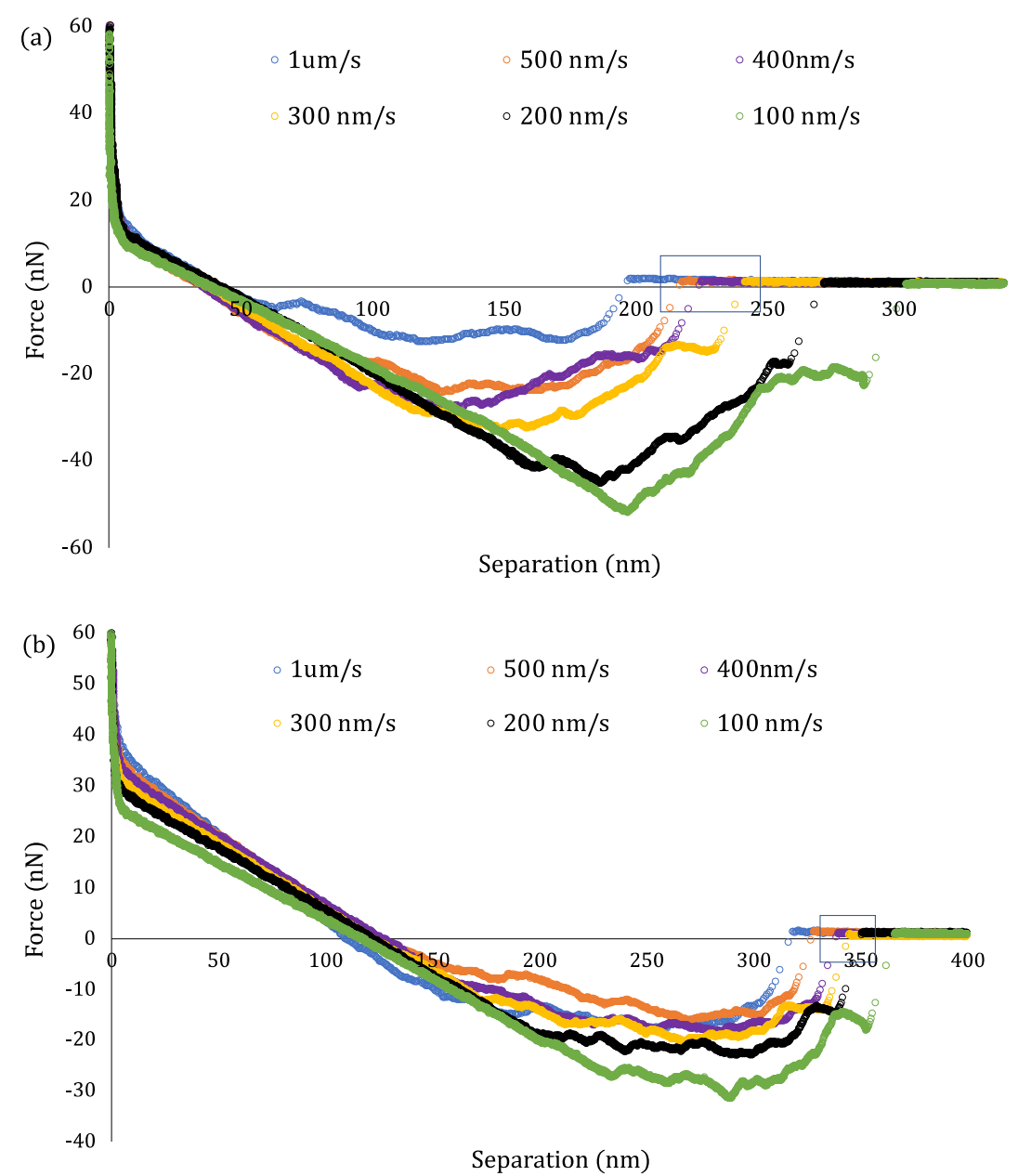

Figure S8. Determining silicone oil film thickness via colloid probe meniscus force measurements using a range of approach velocities $(1000 \mathrm{~nm} / \mathrm{s}$ to $100 \mathrm{~nm} / \mathrm{s})$. (a) and (b) show two sets of force curves taken over two different locations. The approach rate used is indicated in the legend of each panel. Boxes highlight jump-in positions that are clustered together and are used to obtain an average silicone oil thickness for that location.

In Figure S8 the values of approach rate where there is clustering of jump-in distance are between 500 and $300 \mathrm{~nm} / \mathrm{s}$ in location (a), and $400-200 \mathrm{~nm} / \mathrm{s}$ in location (b). The average value of jump-in distance, and therefore silicone oil film thickness, obtained in (a) at 500 - 300 $\mathrm{nm} / \mathrm{s}$ is $227 \pm 10 \mathrm{~nm}$, and in (b) at $400-200 \mathrm{~nm} / \mathrm{s}$ it is $344 \pm 5 \mathrm{~nm}$ (error is the standard deviation). From this data, it appears that approach rates in the range $500-200 \mathrm{~nm} / \mathrm{s}$ are the most reliable, as they provide the lowest scatter values in the jump-in distance. When the rate 
is increased to $500 \mathrm{~nm}$, the film thickness is underestimated relative to this average by $4.9 \%$ in (a) and by $5.0 \%$ in (b). When the rate is decreased to $100 \mathrm{~nm}$ the film thickness is overestimated relative to this average by $34 \%$ in (a) and by $6.6 \%$ in (b). As already shown in Figure S4(c)-(d), an approximate $10 \%$ error is typical even when obtaining the silicone oil thickness in one location using meniscus force measurements, when driving the colloid probe at the same approach rate. For approach rates of $500 \mathrm{~nm} / \mathrm{s}$, these errors are much smaller than what is achievable with other available techniques and remain reproducible across different samples and different locations on the same sample.

In the main manuscript, the silicone oil film thickness was obtained using approach rates of 500 $\mathrm{nm} / \mathrm{s}$ and $1000 \mathrm{~nm} / \mathrm{s}$. Based on Figure S8, it is possible that we have underestimated the true silicone oil thickness due to the hydrodynamic force by approx. 5\% when collecting meniscus force measurements at the approach rate of $500 \mathrm{~nm} / \mathrm{s}$ and by approx. $10 \%$. at the approach rate of $1000 \mathrm{~nm} / \mathrm{s}$.

Figure S9 shows new values of effective slip length fitted with larger silicone oil thickness values (larger by $5 \%$ and $10 \%$, as mentioned above). The slip length values obtained using the corrected silicone oil film thickness are shown as crosses. The experimental data for which the thickness values were originally obtained at $500 \mathrm{~nm} / \mathrm{s}$ are shown as red crosses and those obtained at $1000 \mathrm{~nm} / \mathrm{s}$ are shown as green crosses. They are plotted together with the data in Figure 3(d) from the main text (black dots), which was obtained with the original values of silicone oil thickness. Figure S9 shows that, with the increased silicone oil thicknesses, an increase in the effective slip length is observed by approximately 6 to $18 \%$ for values collected using silicone oil thickness obtained at approach rates of $500 \mathrm{~nm} / \mathrm{s}$, and 9 to $28 \%$ for those at $1000 \mathrm{~nm} / \mathrm{s}$. Here, the new data points sit approximately within the same slope originally established, albeit with a slightly higher value of effective slip length. 


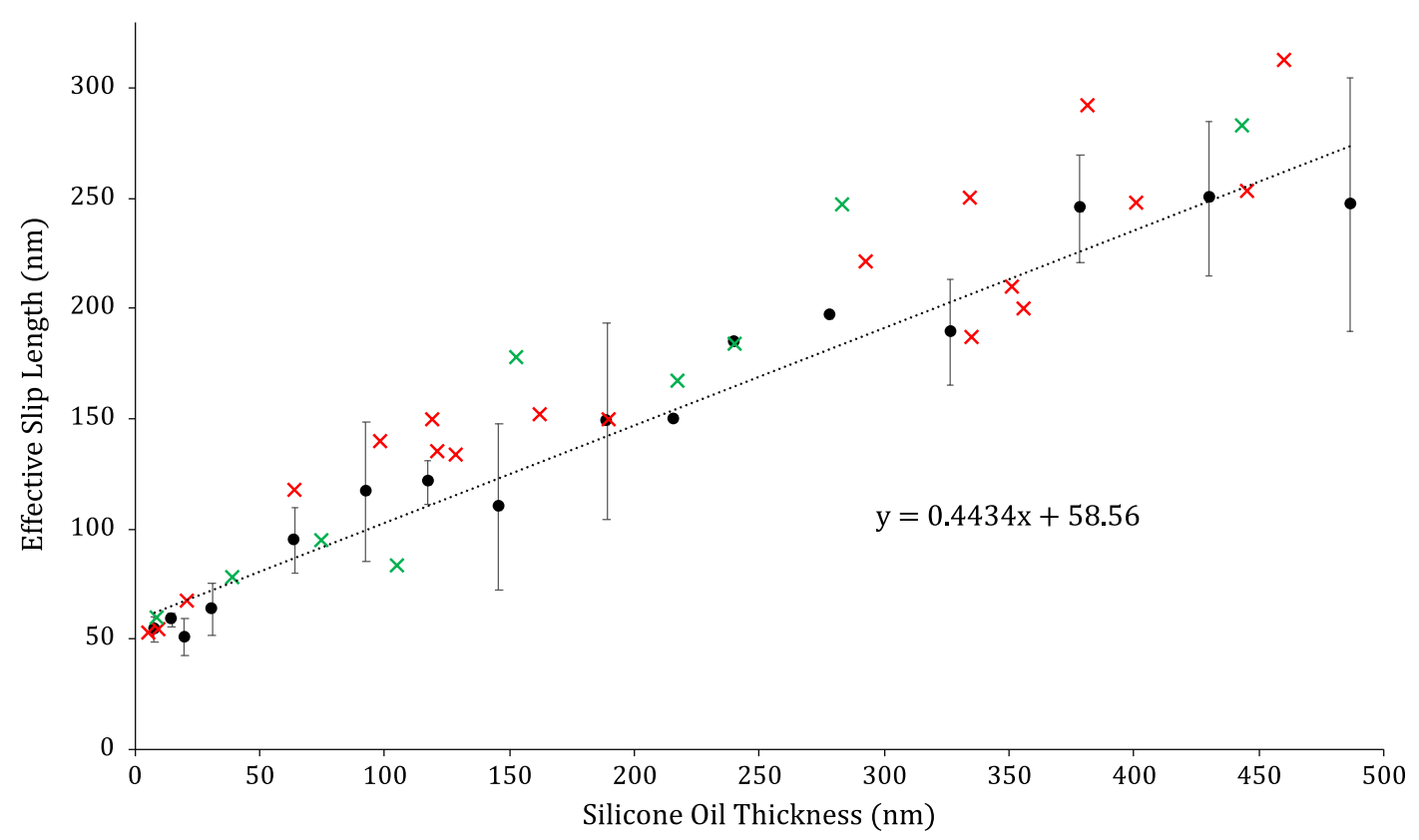

Figure S9. Fitted values of effective slip length versus measured silicone oil film thickness. The black dots were obtained by using silicone oil film thickness obtained at 500 and $1000 \mathrm{~nm} / \mathrm{s}$; red crosses were obtained using a 5\% increase in film thickness relative to the black dots (originally obtained at $500 \mathrm{~nm} / \mathrm{s}$ ) and green crosses were obtained using a 10\% increase in film thickness relative to the black dots at (originally obtained at $1000 \mathrm{~nm} / \mathrm{s}$ ). Error bars are standard deviation from multiple force measurements over similar silicone oil film thicknesses. The straight line is a line of best fit for the black dots.

\section{References}

1. Israelachvili, J. N., Chapter 13 - Van der Waals Forces between Particles and Surfaces. In Intermolecular and Surface Forces (Third Edition), Academic Press: San Diego, 2011; pp 253-289.

2. Israelachvili, J. N., Chapter 6 - Van der Waals Forces. In Intermolecular and Surface Forces (Third Edition), Academic Press: San Diego, 2011; pp 107-132.

3. Rosenberg, B. J.; Van Buren, T.; Fu, M. K.; Smits, A. J., Turbulent drag reduction over air- and liquid-impregnated surfaces. Phys. Fluids 2016, 28 (1), 8.

4. Wexler, J. S.; Jacobi, I.; Stone, H. A., Shear-Driven Failure of Liquid-Infused Surfaces. Phys. Rev. Lett. 2015, 114 (16), 168301. 
5. Fu, M. K.; Arenas, I.; Leonardi, S.; Hultmark, M., Liquid-infused surfaces as a passive method of turbulent drag reduction. J. Fluid Mech. 2017, 824, 688-700.

6. Vinogradova, O. I., Drainage of a Thin Liquid Film Confined between Hydrophobic Surfaces. Langmuir 1995, 11 (6), 2213-2220.

7. Scarratt, L. R. J.; Zhu, L.; Neto, C., How Slippery are SLIPS? Measuring Effective Slip on Lubricated Surfaces with Colloidal Probe Atmoc Force Microscopy. Langmuir 2019, 35 (8), 2976-2982.

8. Zhu, L.; Attard, P.; Neto, C., Reconciling Slip Measurements in Symmetric and Asymmetric Systems. Langmuir 2012, 28 (20), 7768-7774.

9. $\quad$ Peppou-Chapman, S.; Neto, C., Mapping Depletion of Lubricant Films on Antibiofouling Wrinkled Slippery Surfaces. ACS Appl. Mater. Interfaces 2018, 10 (39), 33669-33677. 